18

\title{
Factors Influencing the Formation of Langmuir Films of CdSe/ZnS Colloidal Quantum Dots*
}

\author{
(C) S.A. Savin and A.Yu. Dubavik \\ Department of Optical Physics and Modern Natural Science, ITMO University, \\ 197101 St. Petersburg, Russia
}

e-mail: savin.sergei14@mail.ru

Received July 6, 2018

We prepared thin films containing colloidal quantum dots $\mathrm{CdSe} / \mathrm{ZnS}$ of four different mean core sizes, with photoluminescence maximum in the range $530-610 \mathrm{~nm}$, and covered by different ligands, on the surface of water by the Langmuir method. We obtained Surface pressure - Area curves $(\pi-A$ isotherms) of the formed films at room temperature under different conditions: the compression speed and the waiting time before the film was compressed. In the course of the studies, we established that the type of ligand that passivates the surface of the quantum dots greatly influences the formation of $\mathrm{CdSe} / \mathrm{ZnS}$ Langmuir films, and that the compression speed and the waiting time before the film is compressed do not seriously affect the formation of the films.

DOI: 10.21883/OS.2018.11.46844.220-18

\footnotetext{
* International Conference „PCNSPA 2018 - Photonic Colloidal Nanostructures: Synthesis, Properties, and Applications“, Saint Petersburg, Russia, June 4-8, 2018.

Полный текст статьи опубликован в английской версии журнала.
} 